The FASEB Journal express article 10.1096/fj.04-2415fje. Published online November 17, 2004.

\title{
Molecular profiling of human prostate tissues: insights into gene expression patterns of prostate development during puberty
}

Saravana Mohan Dhanasekaran, ${ }^{*}$ Atreya Dash, ${ }^{\dagger}$ Jianjun Yu, ${ }^{*}$ Ira P. Maine, ${ }^{*}$ Bharathi Laxman, ${ }^{*}$ Scott A. Tomlins, ${ }^{*}$ Chad J. Creighton, ${ }^{\star}$ Anjana Menon, ${ }^{*}$ Mark A. Rubin, ${ }^{\S}$ and Arul M. Chinnaiyan*, ${ }^{\dagger}$

*Department of Pathology, ${ }^{\dagger}$ Michigan Urology Center, ${ }^{*}$ Bioinformatics Program, University of Michigan Medical School, Ann Arbor, Michigan 48109; and ${ }^{\S}$ Department of Pathology, Brigham and Women's Hospital, Dana Farber Cancer Institute, and Harvard Medical School, Boston, Massachusetts 02115

S. M. Dhanasekaran, A. Dash, and J. Yu contributed equally to this work.

Corresponding author: Arul M. Chinnaiyan, M.D., Ph.D., Department of Pathology and Urology, The University of Michigan Medical School, 1301 Catherine Road, MSI Rm. 4237, Ann Arbor, Michigan 48109-0602. E-mail: arul@umich.edu

\section{ABSTRACT}

Testosterone production surges during puberty and orchestrates massive growth and reorganization of the prostate gland, and this glandular architecture is maintained thereafter throughout adulthood. Benign prostatic hyperplasia (BPH) and prostate adenocarcinoma (PCA) are common diseases in adulthood that do not develop in the absence of androgens. Our objective was to gain insight into gene expression changes of the prostate gland at puberty, a crucial juncture in prostate development that is androgen dependent. Understanding the role played by androgens in normal prostate development may provide greater insight into androgen involvement in prostatic diseases. Benign prostate tissues obtained from pubertal and adult age group cadaveric organ donors were harvested and profiled using 20,000 element cDNA microarrays. Statistical analysis of the microarray data identified 375 genes that were differentially expressed in pubertal prostates relative to adult prostates including genes such as Nkx3.1, TMEPAI, TGFBR3, FASN, ANKH, TGFBR2, FAAH, S100P, HoxB13, fibronectin, and TSC2 among others. Comparisons of pubertal and BPH expression profiles revealed a subset of genes that shared the expression pattern between the two groups. In addition, we observed that several genes from this list were previously demonstrated to be regulated by androgen and hence could also be potential in vivo targets of androgen action in the pubertal human prostate. Promoter searches revealed the presence of androgen response elements in a cohort of genes including tumor necrosis factor- $\alpha$ induced adipose related protein, which was found to be induced by androgen. In summary, this is the first report that provides a comprehensive view of the molecular events that occur during puberty in the human prostate and provides a cohort of genes that could be potential in vivo targets of androgenic action during puberty. 
Key words: BPH • microarray • androgen

$\mathrm{D}$ uring prenatal development, the prostate gland falls into a quiescent phase at the third trimester of pregnancy due to a drop in testosterone levels. This dormant phase persists until puberty when the testosterone level surges and the prostate doubles in size and acquires the ability to produce secretions (1). The prostate undergoes dramatic morphologic changes as the epithelial cells proliferate and change from stratified to cuboidal, while glands branch and acini spread, giving rise to the complex glandular architecture seen in the adult prostate. The morphologic and functional changes follow complex reprogramming of the prostate cells that involves alterations of the cytoskeleton and extracellular matrix and changes in downstream signaling pathways that are poorly understood $(2,3)$. Normal puberty is associated with the onset and progressive activation of the hypothalamic-pitutary-gonadal axis and the resultant development of secondary sexual characteristics. This is achieved by increases in gonadotropin releasing hormone $(\mathrm{GnRH})$, luteinizing hormone $(\mathrm{LH})$, and the sex steroids testosterone and dihydrotestosterone (DHT), to control a wide variety of processes during puberty including prostate development. Androgens are important regulators of male sexual differentiation, including their essential role in directing the development of male accessory sex organs such as the prostate $(4,5)$. Androgen binding to its receptor leads to the assembly of activated transcriptional complexes at androgen response elements (AREs) of target gene promoters under the tight regulation of co-activators and co-repressors (6). Understanding the gene expression pattern of the androgen-dependent changes in the normal human prostate until now has largely been dependent on histologic studies (7). cDNA microarray analysis can be used to study the expression of thousands of genes simultaneously and is an important tool to advance biological discovery (8). Three recent studies examined the temporal changes in the global gene expression profile in prostate cancer derived cell lines using cDNA microarrays in response to the synthetic androgen R1881 and or DHT (9-11). These studies identified numerous genes regulated by androgens in androgen responsive human prostate epithelial cancer cell lines. The androgen response in human prostate stromal cells and penile foreskin fibroblasts was also analyzed (12). These studies have been limited in their scope due to use of cell culture models.

The effects of androgens have been extensively studied in prostate models because of the relationship between androgens and the development of widely prevalent neoplastic diseases of the prostate such as benign prostatic hyperplasia (BPH) and prostate adenocarcinoma (PCA). Metastatic PCA regresses with inhibition of androgen production and or blockade of the androgen receptor until androgen-independent transformation occurs. Within the prostate, the enzyme 5- $\alpha$ reductase converts testosterone to the more potent DHT. Inhibition of this activity of the enzyme by the drug finasteride has been shown to decrease the symptoms related to BPH $(13,14)$. Interestingly, finasteride has recently been implicated as a drug that may possibly prevent the development of PCA (15).

Our objective was to characterize the temporal gene expression pattern of the normal human prostate during puberty in a global fashion using cDNA microarrays. This important stage of prostate development occurring during puberty is controlled by the key hormones testosterone and DHT. By identifying the targets of androgen action in vivo in the normal developing prostate, we may gain insight into the pathophysiology of common androgen-dependent neoplastic diseases. 


\section{MATERIALS AND METHODS}

\section{Tissue collection and processing}

In this study, whole prostates and bladders were collected from cadaveric organ donors at the time of transplant organ harvest. This resource was chosen because it provided a source of whole intact specimens that could be processed quickly enough to perform RNA-based studies. The study proceeded with the approval of the Institutional Review Board and the Michigan Transplantation Society. Between July 2001 and present, the University of Michigan Transplant team harvested organs for cadaveric transplant from nine brain dead male organ donors for whom consent to collect research tissue was authorized. Three of the nine donors had a physical appearance consistent with Tanner stage II or III and were classified as pubertal. The remaining six donors appeared to be Tanner stage $\mathrm{V}$ and were classified as adults. Immediately after harvest of transplantable organs, we retrieved the prostate and bladder intact (approximately within 30$60 \mathrm{~min}$ of aortic clamping) and preserved the specimens on ice until return to our institution ( 10-90 $\mathrm{min})$. The prostates were immediately processed as radical prostatectomy surgical specimens. The majority of prostate tissue was placed in OCT, flash frozen in liquid nitrogen, and stored at $-80^{\circ} \mathrm{C}$ until RNA extraction. RNA was extracted using TRIzol reagent according to the instructions of the manufacturer (Gibco-BRL, Rockville, MD). Integrity of RNA was assessed with denaturing agarose gel electrophoresis. Sections from the transition and peripheral zones were dissected, prepared separately, and hybridized as two specimens from the prostate of the 21 year old. For the purposes of analysis, these were considered as two separate adult specimens. In the remaining cases, a representative section from the mid-gland comprised of both peripheral and transition zone tissues was used. Remaining prostate tissue was formalinfixed and subsequently paraffin embedded. Collecting tissues at the time of organ donation provided the advantage of retrieving whole intact prostate for the sole purpose of our studies. The rapid tissue processing after aortic clamping minimized RNA degradation compared with autopsy-acquired tissue. At the time of harvest, serum samples from the organ donors were collected. These were subsequently used to obtain serum testosterone and PSA measurements using a competitive immunoassay technique (Ortho-Clinical Diagnostics, Rochester, NY).

\section{Microarrays and hybridization}

Microarrays (20K chip) containing sequence verified PCR-amplified human cDNAs representing 15,495 Unigene clusters were manufactured as described previously (16) with minor modifications. The clone information can be obtained from our lab web site (http://www.pathology.med.umich.edu/chinnaiyan/index.html). Protocols for printing and postprocessing of arrays are available (http://www.microarrays.org/protocols.html). cDNA microarray analysis of gene expression was done essentially as described previously (16). Briefly, total RNA isolated from prostate tissues was ensured for quality by denaturing agarose gel electrophoresis. The RNA was then reverse transcribed and labeled with cy5 fluorescent dye. The control human prostate total RNA was obtained from a commercial source (Clontech, Palo Alto, CA) and prepared in a similar fashion and labeled with cy3 fluorescent dye. The labeled products were then mixed and hybridized to a $20 \mathrm{~K}$ cDNA array. The images were flagged and normalized using the Genepix software package (Axon Instruments Inc., Union City, CA). Data were median-centered by arrays and only genes that had expression values in at least $80 \%$ of the samples were used in the analysis. 


\section{Statistical analysis of microarrays}

The normalized data set from each experiment was log transformed, filtered for bad spots, and median centered before it was subjected to statistical analysis of microarrays (SAM) as described previously $(17,18)$. The SAM analysis compared the expression profiles of the pubertal to the adult prostates. The significant genes from the SAM analysis were then clustered by implementing an average linkage hierarchical clustering and the output visualized with Tree View software (19).

\section{Histology and immunohistochemistry}

Paraffin embedded prostate tissue blocks were sectioned and stained with hematoxylin and eosin (H\&E). Immunohistochemical staining was performed with polyclonal goat anti-androgen receptor antibody (Santa Cruz Biotechnology, Santa Cruz, CA) at 1:1000 dilution using standard indirect biotin-avidin technique. Slides were reviewed by an experienced genito-urinary pathologist (MAR).

\section{Real-time quantitative PCR and RT-PCR}

To validate microarray results, we performed quantitative real-time PCR (QPCR) for S100P and TMEPAI expression using SYBR green as described previously (20). Briefly, total RNA was reverse transcribed into first-strand cDNA. The quantity of cDNA in each sample was calculated by interpolating its $\mathrm{Ct}$ value vs. a standard curve of $\mathrm{Ct}$ values obtained from serially diluted cDNA from commercially available pooled normal prostate samples (Clontech) and one of the prostate cancer samples. The calculated quantity of S100P or TMEPAI for each sample was then divided by the quantity of the housekeeping gene glyceraldehyde-3 phosphate dehydrogenase (GAPDH) corresponding to each sample to give a relative expression of S100P or TMEPAI for each sample. Primer sequences $5^{\prime}-3^{\prime}$ were as follows: TMEPAI forward: CCTTCTCTTCCCCTTTCCATCTCC, TMEPAI reverse: GTCCCGCCAACCCCAAATCTATCT; S100P forward: CGAGGGCAGCACGCAGACC, S100P reverse: GAGCAATTTATCCACGGCATCCTT. GAPDH primers were as described previously (21). Another control gene hydroxymethylbilane synthase (HMBS) showed a similar pattern to GAPDH expression (data not shown). RT-PCR was performed as described previously (17). The primer sequences for TIARP are as follows: TIARP forward: AGCAAAGCCAAGCAAAGAGTGATG, TIARP reverse: CAGCAGACAAATAGAAGGGGAACC. The amplification was carried out for 28 cycles at $55^{\circ} \mathrm{C}$ as the annealing temperature.

\section{Meta-analysis}

\section{Androgen data set}

The microarray data set for androgen-treated LNCaP cells described by DePrimo et al. (11) was downloaded from Stanford Microarray Database. We selected $9791 \mathrm{mRNA}$ transcripts that had data of sufficient quality (regression correlation $>0.6$ ) in at least three of the four androgen profiles and in at least one of the ethanol profiles. Transcripts in the $\mathrm{LNCaP}$ data set were then ranked, from highest to lowest, by their average ratios across the androgen profiles, minus their average across the ethanol control profiles. Selecting 18,144 transcripts from our profile data set of pubertal and adult prostate tissues with quality data in at least two of the three pubertal 
profiles, we ranked these transcripts, from highest to lowest, by their average ratios across the adult profiles minus the average ratio across the pubertal prostate profiles. The two ranked gene lists (androgen treatment and adult vs. pubertal prostate) shared 8752 transcripts delineated by common unique clone identifiers. The significance of shared overlap between the genes most highly induced by androgen in LNCaP cells and genes most highly up-regulated in adult over pubertal prostates was assessed using the one-sided Fisher's exact test, where the top genes from each ranked gene list ranged from 1 to 1000 .

\section{BPH data set}

From the list of pubertal signature genes from SAM analysis, we selected genes that were differentially expressed in our BPH samples $(16,22)$. We performed two sample $t$ test between $\mathrm{BPH}$ and normal adult group. The genes were then tested on microarray data of Luo et al. (23, 24) using clone ID as cross-reference. For the data set of Luo et al., genes that had expression values in $<80 \%$ of the samples were excluded.

\section{Identification of ARE motifs}

Reference sequences of 360 genes were obtained from the Ref_Seq database at NCBI and used to query the UCSC genome assemblies (http://genome.ucsc.edu/), July 2003 (Human). Each $2 \mathrm{~kb}$ upstream sequence was scanned with the predefined high-quality ARE matrix by using MATCH program integrated in TRANSFAC Professional Suite (http://www.cognia.com). The matched sequence elements with false positive $<0.1$ were reported for each gene. If more than one putative element was identified, the motif mapping nearest to the $5^{\prime}$ end of the reference sequence was selected. All of the putative ARE motifs were aligned by using the CLUSTALW program (http://www.ebi.ac.uk/clustalw/). The sequence logos representing the ARE consensus sequences were generated by the online WEBLOGO program (http://weblogo.berkeley.edu).

\section{RESULTS}

\section{The morphology of the pubertal prostate is distinct from adult prostate}

The clinical details of the cadaveric transplant organ donors are given (Table 1). The pubertal group was comprised of three donors, one aged 9 and two aged 13 years. There were external signs of pubertal onset in these donors, and this was assessed by the external appearance of the genitalia and the distribution of body hair at the time of organ harvest and staged according to the Tanner classification (25). The low serum testosterone levels from these donors were consistent with the developmental stage (Table 1). The adult group consisted of six individuals with an age range from 19-57 years. The causes of brain death were sudden and not related to any genitourinary conditions. The sections of prostate tissue were examined for glandular architecture after H\&E staining. Histology identified the pubertal tissues to have fewer and less differentiated glands (Fig. 1A). Less epithelial branching was observed in the pubertal group when compared with the adults (Fig. 1B). As would be expected, the three pubertal donors had low serum PSA levels ( $\underline{\text { Table } 1})$.

Androgen receptor staining (Fig. 1C and $\underline{1 D}$ ) demonstrated that AR is present in both the stromal as well as the epithelial compartments of both groups; however, it exhibited differences in 
cellular localization. The staining in epithelial cells of the pubertal group was more diffuse, probably due to the distribution of this receptor being predominantly cytoplasmic (Fig. 1C), while mainly nuclear in the adults (Fig. $1 D)$. The androgen receptor protein has previously been shown to be expressed in both stromal as well as the epithelial cell types in prostate $(26,27)$ and cellular localization of the unliganded receptor in transfected cell lines was predominantly cytoplasmic (28).

\section{Gene expression profile of the pubertal prostate}

Microarray analysis was performed using total RNA isolated from the frozen prostate sections that had been analyzed by $\mathrm{H} \& \mathrm{E}$ staining. The labeled cDNA probes prepared from each total RNA sample were hybridized to a human $20 \mathrm{~K}$ chip, which is similar to the chip described earlier (16). The normalized composite data set prepared from all hybridizations was used in SAM (18). This analysis (pubertal vs. the adult samples) yielded a list of 375 statistically significant genes, and the genes were also filtered to have a twofold or above difference in value between the two groups. The genes were analyzed using Cluster (19), implementing average linkage hierarchical clustering, and the output was visualized by TreeView (Fig. 2). The genes displayed in red and green colors represent over and underexpression, respectively, of a given gene compared with the employed reference RNA. The pubertal prostate samples formed a separate cluster owing to their gene expression differences from the adult prostate. Of the 375 genes, we found 131 genes to be up-regulated in the pubertal group while 244 were down-regulated. To confirm the gene expression pattern by an independent method, we performed QPCR for two genes. These were S100P, a calcium binding protein (29), and TMEPAI, also known as PMEPAI, a transmembrane androgen-induced protein (30) (Fig. 3). The Q-PCR values showed a pattern similar to microarrays for both genes. Additionally, we tested two genes, CEBPB and CEBPD, which were up- and down-regulated in pubertal tissues, respectively. We confirmed this pattern by semiquantitative RT-PCR (data not shown).

The differentially expressed genes (Fig. 2) could be classified into several functional groups. Genes involved in fatty acid metabolism such as fatty acid synthase (FASN) and fatty acid amide hydrolase (FAAH); stress response genes like thioredoxin reductase, glutathione synthetase, and NDRG3; and growth factors and their receptors like CYR61, VEGF, and TGF- $\beta$ receptors type II and type III (TGFBR2 and TGFBR3). TGF- $\beta$ growth factor and receptor have previously been shown to be down-regulated by androgen and up-regulated by castration in murine prostate (31, 32 ). In the present study, TGFBR 2 and TGFBR 3 were up-regulated in the pubertal prostates. To better understand the contribution of the stromal fibroblasts to the expresssion profile of the pubertal prostates, we compared our data to a recent study documenting the gene expression signature of androgen receptor dependent fibroblasts derived from urogenital mesenchyme (12). The comparison revealed that genes such as laminin A4, T-box5,3, fibronectin, nidogen, aldehyde dehydrogenase, TIMP1, tenascin (TNXB), fibulin, platelet derived growth factor- $\alpha$ (PDGFRA), and SPARC were highly expressed in genital foreskin fibroblasts and the pubertal prostates. Therefore, the presence of these genes could have reflected the stromal contribution to the expression profile of the pubertal prostates. Genes such as EPS8, S100P, LPL, Mal, Crystallin gamma, ITM2A, and $\alpha-2$ macroglobulin (A2M) were found be underexpressed in the fibroblasts (12) but were highly expressed in the pubertal prostate, implying that the presence of these genes within the pubertal prostates was a contribution from the epithelial compartment. 


\section{Androgen-regulated genes in pubertal prostate}

Several studies have documented the transcriptional regulation of various genes mediated by androgen using DNA microarrays including our own unpublished data (9-11). The statistically significant genes in the SAM analysis were compared with the published data of androgen responsive genes (ARGs). Several of these were found to be common (Table 2). Genes such as TMEPAI, NKx3.1, FASN, and ankylosis homologue (ANKH) have been previously confirmed as ARGs by independent studies using different techniques $(9,10,30,33)$ and were underexpressed in pubertal prostate. Putative AREs have been identified in the promoter regions of these genes while genes like S100P, lipoprotein lipase (LPL), crystallin gamma (CRYG), $\mathrm{A} 2 \mathrm{M}$ and clusterin were known androgen-repressed genes and were found to be up-regulated in the pubertal samples. We compared our pubertal data set with the available androgen-induced gene expression study performed on LNCaP cells (11). A statistically significant correspondence exists (Fig. $4 A$ and $\underline{4 B}$ ) between the genes induced by androgen in the cell line and genes underexpressed in pubertal prostate tissues $(P<10 \mathrm{E}-25$, by Fisher's exact, for the overlap between the top 500 genes from each data set) demonstrating the in vitro system of androgen induction as a good model for in vivo behavior. A list of the top 40 genes that were both underexpressed in pubertal and overexpressed in $\mathrm{LNCaP}$ cells treated with androgen is displayed (Fig. 4C). In addition, we searched up to $2 \mathrm{~Kb}$ upstream of the transcription start site for 360 reference sequences corresponding to the significant genes from our analysis with a predefined high-quality ARE matrix by using the MATCH program integrated in TRANSFAC Professional Suite. The program identified promoters of 52 genes to possess a putative ARE. The alignment of identified AREs and the consensus ARE sequence is shown (Fig. 5). A few of these genes were tested for androgen regulation by performing RT-PCR with gene specific primers on RNA isolated from the LNCaP prostate cell line treated with the synthetic androgen R1881 along a time course. The gene TMEPAI, present among the shortlisted genes from our study and previously demonstrated to be up-regulated by androgen, was used as a positive control for androgen response in our system. Primers designed for TIARP amplified a specific product and revealed it also to be up-regulated by androgen in treated $\mathrm{LnCaP}$ cells (Fig. 5). A TNF- $\alpha$ inducible 470 amino acid protein termed TIARP has six transmembrane regions, a large $\mathrm{N}$

terminus, and a short carboxy-terminal tail, a structure reminiscent of channel and transporter proteins. TIARP has been hypothesized to play a role in adipocyte development and metabolism (34).

\section{BPH and other prostate disease states}

Corresponding expression values for the 375 SAM selected genes were queried against our prostate cancer $20 \mathrm{~K}$ cDNA microarray data set (www.oncomine.org) This data set consisted of $19 \mathrm{BPH}, 23$ localized PCA, and 8 metastatic prostate cancer (MET) samples. We found three regions of similarity between the pubertal and BPH samples (Fig. 6), two of which were upregulated (Fig. 6A and $\underline{6 B}$ ) and one down-regulated (Fig. 6C). The subset of common upregulated genes included TGFBR2 and TGFBR3. IGF II, which is synthesized and secreted by stroma (35), was found to be up-regulated in pubertal prostates. Expression of laminin $\beta 1$ has been localized to basal cells (36). To further explore the gene expression similarity observed between pubertal and BPH samples, we compared pubertal signature genes to our BPH data set (present study) and an independent BPH gene expression data set previously reported by Luo et al. $(23,24)$ (Fig. 7). Among the 375 statistically significant genes from SAM analysis, we found 
197 genes to be differentially expressed in our BPH samples compared with normal adult tissues by two sample $t$ test $(\mathrm{P}<0.05$, one-side). These 197 genes were then tested on data set of Luo et al. $(23,24)$ using clone ID as cross-reference. We found that 73 out of 129 available genes in their data were also differentially expressed in the nine BPH samples they profiled (against 25 normal samples, one-sided $t$ test, $P<0.05$ ). Laminin A4 and A2M, laminin $\beta 1$, and epidermal growth factor receptor substrate 8 (EPS8) are up-regulated in pubertal prostate and have been shown to be overexpressed in our BPH data set and the previously reported BPH microarray profile $(23,24)$. Previously demonstrated androgen-regulated genes like VEGF, TPD52, and ANKH were underexpressed in pubertal prostates and BPH specimens.

\section{DISCUSSION}

We examined gene expression changes occurring during human prostate development at puberty by comparing microarray analyses from pubertal and adult prostates. This revealed that pubertal prostates possessed a distinct gene expression signature. This unique gene expression signature could be attributed to several characteristics of the prostate during puberty. The gene expression differences observed between the pubertal and adult prostate may account for the difference in cell type representation between the two stages of prostate. Shapiro et al. (7) has previously reported the changes in cellular composition of the prostate during various stages of development. They described inversely related age-dependent change in smooth muscle and connective tissue. Although there was a progressive increase in connective tissue until puberty and a decline thereafter, the smooth muscle declined thoroughout childhood until puberty and increased in adolescence and early adulthood. Morphologically there was a lower smooth muscle to connective tissue ratio within the stromal compartment of pubertal prostate compared with the adults (7). However, the stromal to epithelial ratio remained constant. Hence, one of the major factors influencing this gene expression profile could be the difference in cell type representation between the two stages.

The second observation that we made was the link between androgen regulation of various genes and their expression pattern in the pubertal prostate. We confirmed that the pubertal prostates had low testosterone levels consistent with the physical appearance of the gland. Interestingly, we found high expression of S100P, crystallin gamma, and clusterin in pubertal prostates, which were described previously as androgen-suppressed genes. In addition, we found genes such as TMEPAI, NKx3.1, LPL, GLUD, IQGAP2, ANKH, and TPD52 were underexpressed in pubertal prostates, which were demonstrated in multiple previous studies to be up-regulated by androgen. Therefore, we hypothesized that amongst the pubertal prostate signature genes, a subset of genes could be potential in vivo targets of androgen. Recent work from our group suggests that TPD52 is both amplified and androgen regulated in cancer but not the normal state (37). Studies revealed that androgen has a role in expression of the stress response genes in the prostate cancer cell lines (10). We observed that stress response genes such as thioredoxin reductase, glutathione synthetase, and NRDG3 were down-regulated in the pubertal prostate compared with adult prostates, indirectly raising the possibility for a role in the stress response for androgen. We also analyzed the promoters for 375 genes up to $3 \mathrm{~Kb}$, in search of putative ARE. We identified 52 genes that had one or more AREs in their promoter sequence. We tested a few genes for androgen regulation in LNCap cells. TMEPAI was used as a positive control in RT-PCRs, and it showed an induction of its message with androgen treatment as reported earlier. TIARP/FLJ23153, a six transmembrane protein that was selected for RT-PCR analysis, was also 
induced by androgen. This transcriptional control unknown previously for TIARP is reported here for the first time. It is noteworthy that a previous study of androgen receptor knockout (ARKO) mice demonstrated a decrease in number and size of adipocytes in mice lacking the androgen receptor. Hence our finding of TIARP induction by androgen and the earlier observation from ARKO mice imply a role for TIARP in androgen-mediated adipoctye function and development (5). We suggest that TIARP plays a role in androgen-mediated action at least in adipocytes. Further studies are required to understand the role of TIARP in the prostate and adipocyte. An intriguing recent finding demonstrated that factors secreted by adipocytes, including leptin can stimulate prostate cancer cell line proliferation (38).

In our study, Hox B6 and Hox B13 were up- and down-regulated respectively in the pubertal prostates. Hox gene expression in development and neoplastic conditions of the prostate has been reported (4, 39, 40). Among the Hox family genes Hox A10, A13, B13, and D13 have been shown to be required for normal prostate morphogenesis in murine models. A double knockout of Hox B13 and D13 results in a severe defect in mouse ventral prostate development. Immunohistochemical staining against Hox B13 and D13 proteins showed the expression to be restricted to the epithelial compartment (41-43). Neural cell adhesion molecule 1 (NCAM1) has been identified as a target of Hox genes (44). In particular, studies in cell lines have shown that Hox C6 and Hox B9 increased NCAM1 promoter activity. In our study, pubertal tissues had an elevated expression of NCAM1, consistent with Hox-related prostate development in humans. We speculate that the stages of prostate development are related to the temporal expression of particular Hox genes.

Nkx3.1 is a homeobox gene that has given rise to interest in prostate development as well as prostate cancer $(45,46)$. In murine studies, Nkx3.1 initiated prostate epithelial development as well as ductal outgrowth of epithelial glands and subsequent regulation of epithelial growth and secretory function. Interestingly, $\mathrm{Nkx} 3.1$ expression appeared to be one of the earliest responses to androgens (4). In tissue recombination studies, the mode of Nkx3.1 expression in the developing prostate was related to stromal-epithelial interaction. The recombination studies have shown that mesenchymal androgen receptors direct epithelial branching though paracrine action. Later in development androgen receptors within the epithelium are necessary for secretory function. In our study, the adult prostates overexpressed Nkx3.1 relative to the pubertal prostates, consistent with the androgen regulation of this gene.

We also compared the pubertal prostate signature to other diseased prostate tissue profiles and observed that a subset of genes had a similar trend in expression between the pubertal and BPH specimens. We further narrowed down the genes in the overlap by including a previously reported BPH profile $(23,24)$ in our analysis. Future detailed analysis of the BPH data set will help in understanding the underlying process that has led to the shared gene expression profile observed between the pubertal and BPH samples. The stromal to epithelial ratio remains constant from birth to age 40 in nonhyperplastic glands and is similar to the ratios in tissues derived from asymptomatic and symptomatic BPH patients. However, there was a difference between pubertal and adult prostates in the percentage of smooth muscle and connective tissue that comprise the stromal compartment (7). Earlier findings suggested a "reawakening" of fetal processes in BPH (47). This was based on the observation that ontogenetic processes are recapitulated in the development of stromal nodules in BPH. In this context, the significance of the genes that contributed to the shared signature between $\mathrm{BPH}$ and pubertal tissues requires further study. 
Stromal-epithelial interactions play an important role in prostate function and development and also in many prostate disease conditions $(48,49)$. Thus, orchestrated gene expression cascades that can be regulated by androgen may occur simultaneously in both the compartments during development. Several genes identified by this study (Fig. 2) may also play an important role in the stromal-epithelial interactions in prostate during puberty. The pubertal prostate samples that we analyzed were derived from donors whose testosterone had not yet reached mature adult levels to fully activate the androgen-dependent cascade.

In summary, this study describes the transcriptome signature of the pubertal prostate. The observed gene expression changes may have derived from both the stromal and epithelial compartments. Many of the genes with altered expression during the pubertal process were also shown to be regulated by androgen. Further characterization of genes from this list could identify novel in vivo targets of androgen in the human prostate. Future studies will be aimed at resolving the role of shortlisted genes identified in this study and their complex relationships between androgen action and development.

Supplementary information (DNA microarray data sets) will be available at the author's web site: http://chinnaiyan.path.med.umich.edu/.

\section{ACKNOWLEDGMENTS}

We thank Michelle Leblanc for sectioning and immunohistochemistry, Ronglai Shen for helping with figures and Dr. James Varani for helpful suggestions. We thank the University of Michigan, Section of Transplant Surgery; the Transplantation Society of Michigan; and the University of Michigan Survival Flight for their assistance in retrieval of prostate tissue. S. M. Dhanasekaran is sponsored by the grant from Department of Defense, grant PC020655. The following grants supported this work: MTOPS Prostate Sample Analysis Consortium (MPSA) (U01 AG22312) and NIH P50CA69568.

\section{REFERENCES}

1. Donjacour, A. A., and Cunha, G. R. (1988) The effect of androgen deprivation on branching morphogenesis in the mouse prostate. Dev. Biol. 128, 1-14

2. Aumuller, G., and Seitz, J. (1990) Protein secretion and secretory processes in male accessory sex glands. Int. Rev. Cytol. 121, 127-231

3. Partin, A. W. R. R. (2002) The Molecular Biology, Endocrinology, and Physiology of the Prostate and Seminal Vesicles, Saunders, Philadelphia, PA

4. Marker, P. C., Donjacour, A. A., Dahiya, R., and Cunha, G. R. (2003) Hormonal, cellular, and molecular control of prostatic development. Dev. Biol. 253, 165-174

5. Yeh, S., Tsai, M. Y., Xu, Q., Mu, X. M., Lardy, H., Huang, K. E., Lin, H., Yeh, S. D., Altuwaijri, S., Zhou, X., et al. (2002) Generation and characterization of androgen receptor knockout (ARKO) mice: an in vivo model for the study of androgen functions in selective tissues. Proc. Natl. Acad. Sci. USA. 99, 13498-13503 
6. Gelmann, E. P. (2002) Molecular biology of the androgen receptor. J. Clin. Oncol. 20, 3001-3015

7. Shapiro, E., Hartanto, V., Perlman, E. J., Tang, R., Wang, B., and Lepor, H. (1997) Morphometric analysis of pediatric and nonhyperplastic prostate glands: evidence that BPH is not a unique stromal process. Prostate 33, 177-182

8. Schena, M., Shalon, D., Davis, R. W., and Brown, P. O. (1995) Quantitative monitoring of gene expression patterns with a complementary DNA microarray. Science 270, 467-470

9. Nelson, P. S., Clegg, N., Arnold, H., Ferguson, C., Bonham, M., White, J., Hood, L., and Lin, B. (2002) The program of androgen-responsive genes in neoplastic prostate epithelium. Proc. Natl. Acad. Sci. USA. 99, 11890-11895

10. Segawa, T., Nau, M. E., Xu, L. L., Chilukuri, R. N., Makarem, M., Zhang, W., Petrovics, G., Sesterhenn, I. A., McLeod, D. G., Moul, J. W., et al. (2002) Androgen-induced expression of endoplasmic reticulum (ER) stress response genes in prostate cancer cells. Oncogene 21, 8749-8758

11. DePrimo, S. E., Diehn, M., Nelson, J. B., Reiter, R. E., Matese, J., Fero, M., Tibshirani, R., Brown, P. O., and Brooks, J. D. (2002) Transcriptional programs activated by exposure of human prostate cancer cells to androgen. Genome Biol. 3, RESEARCH0032

12. Holterhus, P. M., Hiort, O., Demeter, J., Brown, P. O., and Brooks, J. D. (2003) Differential gene-expression patterns in genital fibroblasts of normal males and 46,XY females with androgen insensitivity syndrome: evidence for early programming involving the androgen receptor. Genome Biol. 4, R37

13. Gormley, G. J., Stoner, E., Bruskewitz, R. C., Imperato-McGinley, J., Walsh, P. C., McConnell, J. D., Andriole, G. L., Geller, J., Bracken, B. R., Tenover, J. S., et al. (1992) The effect of finasteride in men with benign prostatic hyperplasia. The Finasteride Study Group. N. Engl. J. Med. 327, 1185-1191

14. Bruskewitz, R., Girman, C. J., Fowler, J., Rigby, O. F., Sullivan, M., Bracken, R. B., Fusilier, H. A., Kozlowski, D., Kantor, S. D., Johnson, E. L., et al. (1999) Effect of finasteride on bother and other health-related quality of life aspects associated with benign prostatic hyperplasia. PLESS Study Group. Proscar Long-term Efficacy and Safety Study. Urology 54, 670-678

15. Thompson, I. M., Goodman, P. J., Tangen, C. M., Lucia, M. S., Miller, G. J., Ford, L. G., Lieber, M. M., Cespedes, R. D., Atkins, J. N., Lippman, S. M., et al. (2003) The influence of finasteride on the development of prostate cancer. N. Engl. J. Med. 349, 215-224

16. Dhanasekaran, S. M., Barrette, T. R., Ghosh, D., Shah, R., Varambally, S., Kurachi, K., Pienta, K. J., Rubin, M. A., and Chinnaiyan, A. M. (2001) Delineation of prognostic biomarkers in prostate cancer. Nature 412, 822-826 
17. Varambally, S., Dhanasekaran, S. M., Zhou, M., Barrette, T. R., Kumar-Sinha, C., Sanda, M. G., Ghosh, D., Pienta, K. J., Sewalt, R. G., Otte, A. P., et al. (2002) The polycomb group protein EZH2 is involved in progression of prostate cancer. Nature 419, 624-629

18. Tusher, V. G., Tibshirani, R., and Chu, G. (2001) Significance analysis of microarrays applied to the ionizing radiation response. Proc. Natl. Acad. Sci. USA. 98, 5116-5121

19. Eisen, M. B., Spellman, P. T., Brown, P. O., and Botstein, D. (1998) Cluster analysis and display of genome-wide expression patterns. Proc. Natl. Acad. Sci. USA 95, 14863-14868

20. Kleer, C. G., Cao, Q., Varambally, S., Shen, R., Ota, I., Tomlins, S. A., Ghosh, D., Sewalt, R. G., Otte, A. P., Hayes, D. F., et al. (2003) EZH2 is a marker of aggressive breast cancer and promotes neoplastic transformation of breast epithelial cells. Proc. Natl. Acad. Sci. USA. 100, 11606-11611

21. Vandesompele, J., De Preter, K., Pattyn, F., Poppe, B., Van Roy, N., De Paepe, A., and Speleman, F. (2002) Accurate normalization of real-time quantitative RT-PCR data by geometric averaging of multiple internal control genes. Genome Biol. 3, RESEARCH0034

22. Rhodes, D. R., Yu, J., Shanker, K., Deshpande, N., Varambally, R., Ghosh, D., Barrette, T., Pandey, A., and Chinnaiyan, A. M. (2004) ONCOMINE: a cancer microarray database and integrated data-mining platform. Neoplasia 6, 1-6

23. Luo, J., Dunn, T., Ewing, C., Sauvageot, J., Chen, Y., Trent, J., and Isaacs, W. (2002) Gene expression signature of benign prostatic hyperplasia revealed by cDNA microarray analysis. Prostate 51, 189-200

24. Luo, J., Dunn, T. A., Ewing, C. M., Walsh, P. C., and Isaacs, W. B. (2003) Decreased gene expression of steroid 5 alpha-reductase 2 in human prostate cancer: implications for finasteride therapy of prostate carcinoma. Prostate 57, 134-139

25. Hiort, O. (2002) Androgens and puberty. Best Pract. Res. Clin. Endocrinol Metab. 16, 3141

26. Gnanapragasam, V. J., Robson, C. N., Leung, H. Y., and Neal, D. E. (2000) Androgen receptor signalling in the prostate. BJU Int. 86, 1001-1013

27. Aumuller, G., Holterhus, P. M., Konrad, L., von Rahden, B., Hiort, O., Esquenet, M., and Verhoeven, G. (1998) Immunohistochemistry and in situ hybridization of the androgen receptor in the developing human prostate. Anat. Embryol. (Berl.) 197, 199-208

28. Simental, J. A., Sar, M., Lane, M. V., French, F. S., and Wilson, E. M. (1991) Transcriptional activation and nuclear targeting signals of the human androgen receptor. $J$. Biol. Chem. 266, 510-518

29. Averboukh, L., Liang, P., Kantoff, P. W., and Pardee, A. B. (1996) Regulation of S100P expression by androgen. Prostate 29, 350-355 
30. Xu, L. L., Su, Y. P., Labiche, R., Segawa, T., Shanmugam, N., McLeod, D. G., Moul, J. W., and Srivastava, S. (2001) Quantitative expression profile of androgen-regulated genes in prostate cancer cells and identification of prostate-specific genes. Int. J. Cancer 92, 322-328

31. Kyprianou, N., and Isaacs, J. T. (1989) Expression of transforming growth factor-beta in the rat ventral prostate during castration-induced programmed cell death. Mol. Endocrinol. 3, $1515-1522$

32. Kim, I. Y., Ahn, H. J., Zelner, D. J., Park, L., Sensibar, J. A., and Lee, C. (1996) Expression and localization of transforming growth factor-beta receptors type I and type II in the rat ventral prostate during regression. Mol. Endocrinol. 10, 107-115

33. Xu, L. L., Shanmugam, N., Segawa, T., Sesterhenn, I. A., McLeod, D. G., Moul, J. W., and Srivastava, S. (2000) A novel androgen-regulated gene, PMEPA1, located on chromosome 20 q13 exhibits high level expression in prostate. Genomics 66, 257-263

34. Moldes, M., Lasnier, F., Gauthereau, X., Klein, C., Pairault, J., Feve, B., and ChambautGuerin, A. M. (2001) Tumor necrosis factor-alpha-induced adipose-related protein (TIARP), a cell-surface protein that is highly induced by tumor necrosis factor-alpha and adipose conversion. J. Biol. Chem. 276, 33938-33946

35. Peehl, D. M., Cohen, P., and Rosenfeld, R. G. (1995) The insulin-like growth factor system in the prostate. World J. Urol. 13, 306-311

36. Pfohler, C., Fixemer, T., Jung, V., Dooley, S., Remberger, K., and Bonkhoff, H. (1998) In situ hybridization analysis of genes coding collagen IV alphal chain, laminin betal chain, and S-laminin in prostate tissue and prostate cancer: increased basement membrane gene expression in high-grade and metastatic lesions. Prostate 36, 143-150

37. Rubin, M. A., Varambally, S., Beroukhim, R., Tomlins, S. A., Rhodes, D. R., Paris, P. L., Hofer, M. D., Storz-Schweizer, M., Kuefer, R., Fletcher, J. A., et al. (2004) Overexpression, amplification, and androgen regulation of TPD52 in prostate cancer. Cancer Res. 64, 38143822

38. Onuma, M., Bub, J. D., Rummel, T. L., and Iwamoto, Y. (2003) Prostate cancer celladipocyte interaction: leptin mediates androgen-independent prostate cancer cell proliferation through c-Jun NH2-terminal kinase. J. Biol. Chem. 278, 42660-42667

39. Miller, G. J., Miller, H. L., van Bokhoven, A., Lambert, J. R., Werahera, P. N., Schirripa, O., Lucia, M. S., and Nordeen, S. K. (2003) Aberrant HOXC expression accompanies the malignant phenotype in human prostate. Cancer Res. 63, 5879-5888

40. Nunes, F. D., de Almeida, F. C., Tucci, R., and de Sousa, S. C. (2003) Homeobox genes: a molecular link between development and cancer. Pesqui. Odontol. Bras. 17, 94-98

41. Podlasek, C. A., Duboule, D., and Bushman, W. (1997) Male accessory sex organ morphogenesis is altered by loss of function of Hoxd-13. Dev. Dyn. 208, 454-465 
42. Podlasek, C. A., Barnett, D. H., Clemens, J. Q., Bak, P. M., and Bushman, W. (1999) Prostate development requires Sonic hedgehog expressed by the urogenital sinus epithelium. Dev. Biol. 209, 28-39

43. Warot, X., Fromental-Ramain, C., Fraulob, V., Chambon, P., and Dolle, P. (1997) Gene dosage-dependent effects of the Hoxa-13 and Hoxd-13 mutations on morphogenesis of the terminal parts of the digestive and urogenital tracts. Development 124, 4781-4791

44. Edelman, G. M., and Jones, F. S. (1995) Developmental control of N-CAM expression by Hox and Pax gene products. Philos. Trans. R. Soc. Lond. B Biol. Sci. 349, 305-312

45. Shen, M. M., and Abate-Shen, C. (2003) Roles of the Nkx3.1 homeobox gene in prostate organogenesis and carcinogenesis. Dev. Dyn. 228, 767-778

46. Bhatia-Gaur, R., Donjacour, A. A., Sciavolino, P. J., Kim, M., Desai, N., Young, P., Norton, C. R., Gridley, T., Cardiff, R. D., Cunha, G. R., et al. (1999) Roles for Nkx3.1 in prostate development and cancer. Genes Dev 13, 966-977

47. Bierhoff, E., Walljasper, U., Hofmann, D., Vogel, J., Wernert, N., and Pfeifer, U. (1997) Morphological analogies of fetal prostate stroma and stromal nodules in BPH. Prostate 31, 234-240

48. Cunha, G. R., Hayward, S. W., and Wang, Y. Z. (2002) Role of stroma in carcinogenesis of the prostate. Differentiation 70, 473-485

49. Farnsworth, W. E. (1999) Prostate stroma: physiology. Prostate 38, 60-72

Received July 7, 2004; accepted October 7, 2004. 
Table 1

Clinical characteristics of organ donors used in this study

\begin{tabular}{|c|c|c|c|c|c|c|}
\hline Donor & Age & Race $^{\text {a }}$ & Cause of Brain Death & Tanner Stage $^{c}$ & $\begin{array}{l}\text { Serum } \\
\text { testosterone } \\
\text { (ng/dl) }\end{array}$ & $\begin{array}{l}\text { Serum } \\
\text { PSA } \\
\text { (ng/dl) }\end{array}$ \\
\hline 1 & 13 & $\mathrm{C}$ & CHI-trauma & III & 0.37 & 0.068 \\
\hline 2 & 9 & AA & ABI-drowning & II & 0.22 & 0.000 \\
\hline 3 & 13 & $\mathrm{C}$ & CHI-trauma & III & 0.24 & 0.198 \\
\hline 4 & 22 & $\mathrm{C}$ & SAH & V & 4.07 & 0.588 \\
\hline 5 & 57 & $\mathrm{C}$ & $\mathrm{ICH}$ & V & 7.04 & 0.845 \\
\hline 6 & 46 & $\mathrm{C}$ & $\mathrm{ICH}$ & V & 0.86 & 0.586 \\
\hline 7 & 21 & AA & GSW & V & 9.07 & 0.422 \\
\hline 8 & 45 & $\mathrm{C}$ & SAH & V & 2.05 & 1.46 \\
\hline 9 & 19 & $\mathrm{C}$ & $\mathrm{ICH}$ & V & 5.73 & 0.434 \\
\hline
\end{tabular}

${ }^{\mathrm{a}}$ Race: Caucasian (C) or African-American (AA). ${ }^{\mathrm{b}}$ Cause of brain death: anoxic brain injury (ABI); closed head injury (CHI); gunshot wound to head (GSW); intracranial hemorrhage (ICH); subarachnoid hemorrhage (SAH). ${ }^{\mathrm{C}}$ Tanner criteria (22). 
Table 2

Expression of androgen regulated genes in pubertal prostate

\begin{tabular}{|c|c|c|c|c|c|}
\hline \multirow[b]{3}{*}{ Gene symbol } & \multicolumn{4}{|c|}{ Microarray data } & \multirow{3}{*}{ Northern data ${ }^{b}$} \\
\hline & \multirow{2}{*}{$\begin{array}{l}\text { Mean fold change } \\
\text { Current study }\end{array}$} & \multicolumn{3}{|c|}{ Reported androgen regulation in cell lines ${ }^{a}$} & \\
\hline & & $\mathbf{A}$ & $\mathbf{B}$ & $\mathbf{C}$ & \\
\hline AZGP1 & -3.1 & & & + & \\
\hline CEBPD & -3.7 & & + & & \\
\hline CHK & -1.9 & & + & & \\
\hline ELL2 & -2.7 & + & + & + & \\
\hline FAAH & -2.2 & & + & & \\
\hline GUCY1A3 & -2.7 & + & + & + & \\
\hline IQGAP2 & -2.0 & + & + & + & \\
\hline LCP1 & -2.6 & + & + & & \\
\hline NKX3-1 & -10.6 & + & + & + & + \\
\hline PDE7A & -2.7 & & + & & \\
\hline SEPP1 & -2.0 & & & + & \\
\hline TMEPAI & -5.4 & + & + & + & + \\
\hline TPD52 & -2.7 & + & & + & + \\
\hline TSC2 & -3.0 & & + & & \\
\hline ANKH & -3.3 & + & + & + & + \\
\hline S100P & 3.8 & & & & - \\
\hline CLU & 2.0 & & & & - \\
\hline LPL & 2.2 & & & & - \\
\hline FN1 & 3.0 & - & & & \\
\hline
\end{tabular}

+: Up-regulation, -: down-regulation; ${ }^{\mathrm{a}}$ refs $9,10,11 ;{ }^{\mathrm{b}}$ refs $9,11,26,30,31$. 
Fig. 1
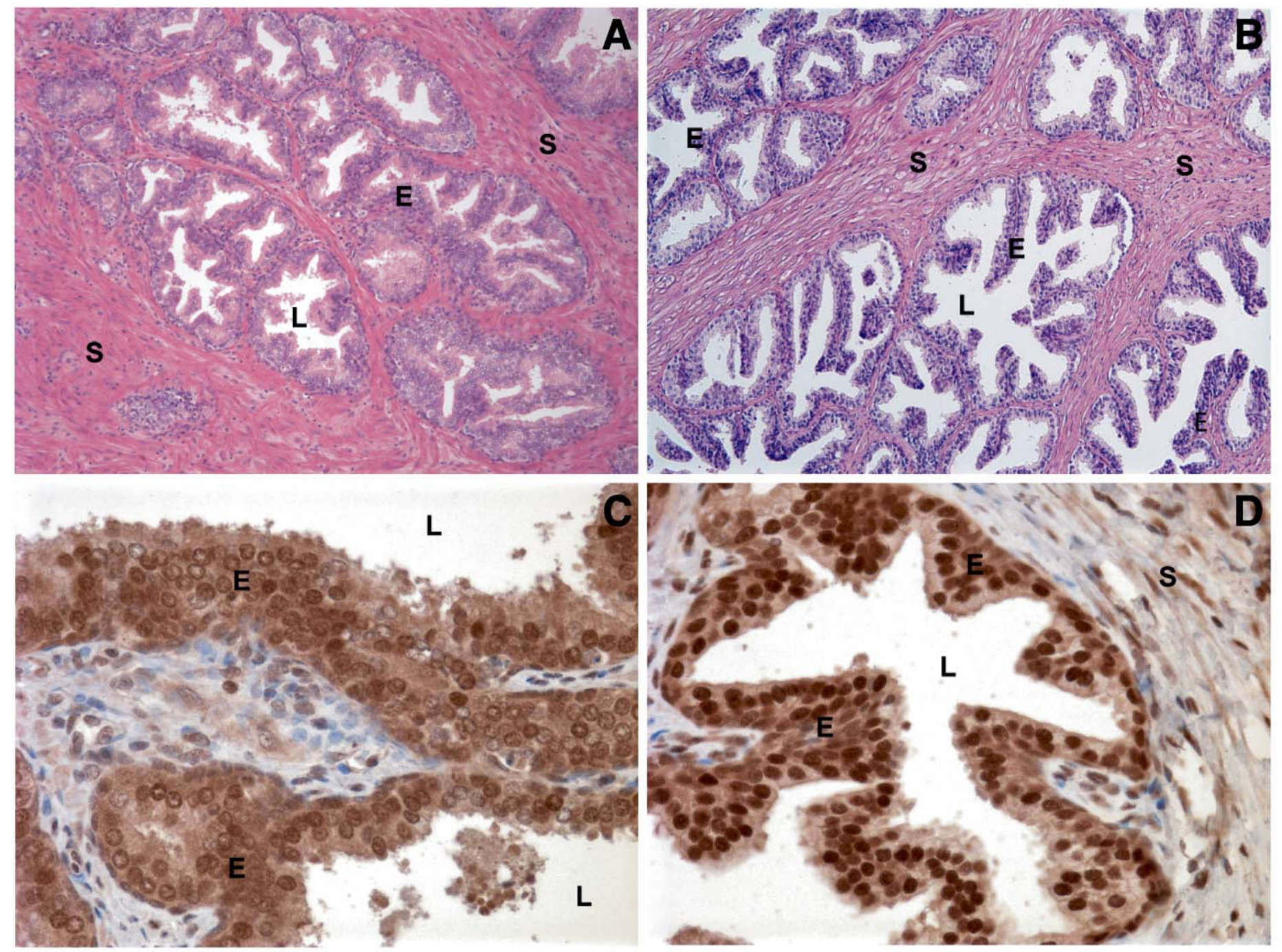

Figure 1. Morphology and androgen receptor expression of pubertal and adult prostate. Histology of a

representative pubertal $(\boldsymbol{A})$ and adult $(\boldsymbol{B})$ prostate tissue, respectively. Sections were stained with H\&E and images were taken at $\times 100$ magnification. Androgen receptor expression as assessed by immunohistochemical staining of pubertal $(\boldsymbol{C})$ and adult $(\boldsymbol{D})$ tissue respectively. Images were captured at $\times 400$ magnification. E: epithelium; S: stroma; L: lumen. 
Fig. 2
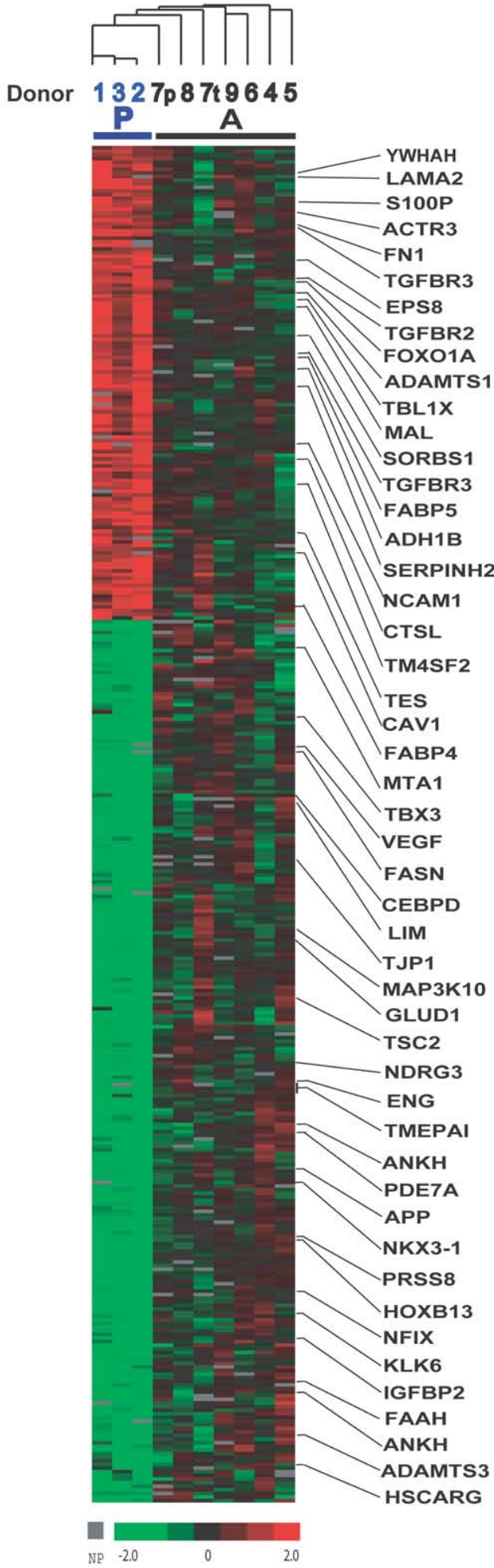

Figure 2. Gene expression profile of pubertal prostate. Pattern of expression for 375 genes selected by 2 class SAM analysis (18), visualized by Tree View (19). Red indicates upregulated genes, green indicates down-regulated genes, black stands for no change, while gray refers to data not available. Donor $7 \mathrm{p}, 7 \mathrm{t}$ stand for peripheral and transitional zones respectively from donor 7 . The gene symbols are provided (HUGO nomenclature). P: pubertal; A: adult. 
Fig. 3

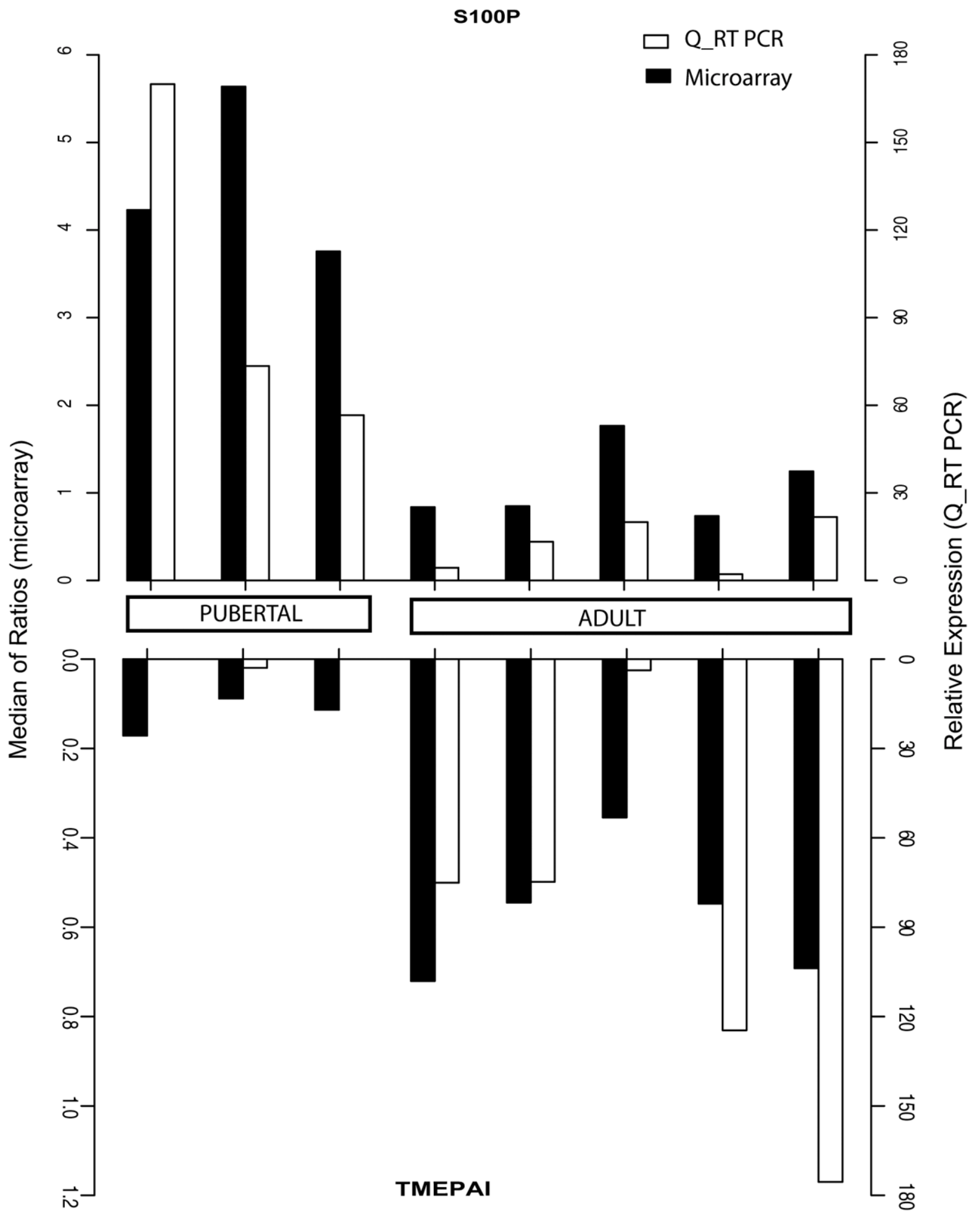

Figure 3. Confirmation of microarray results by quantitative real-time PCR. Normalized median of ratio gene expression values from microarrays (filled bars) are compared with the relative expression values obtained with quantitative real-time PCR (open bars) for the genes S100P (top) and TMEPAI (bottom). 
Fig. 4

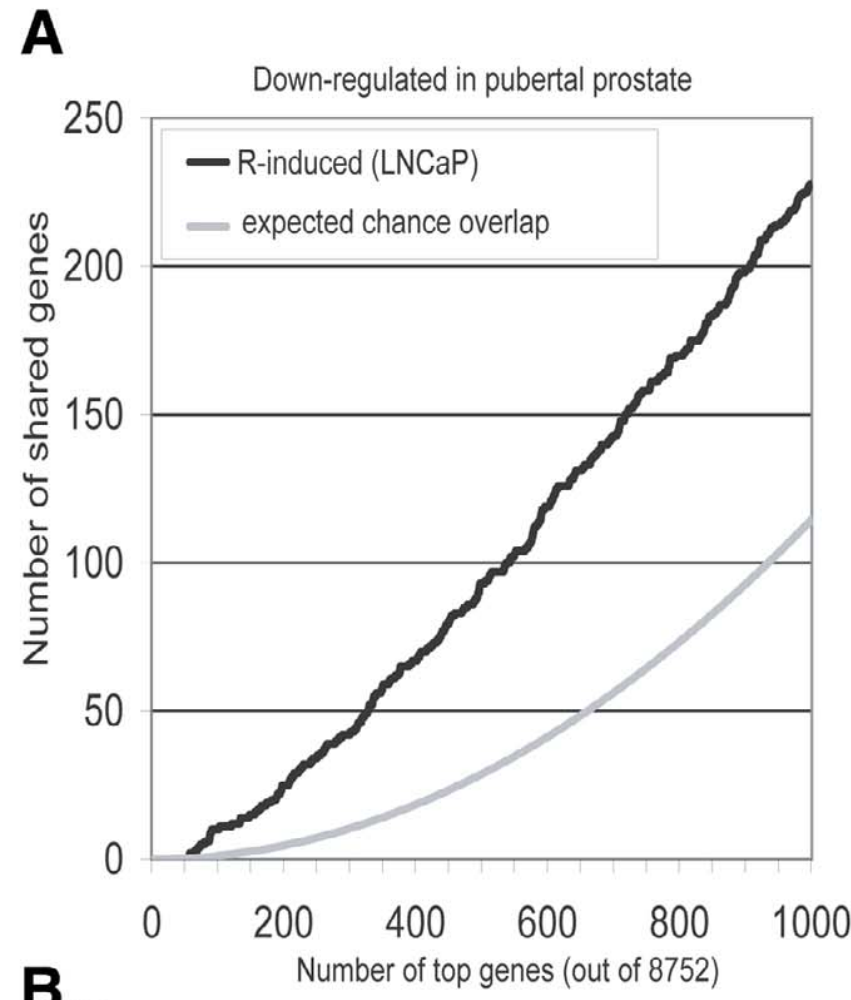

C

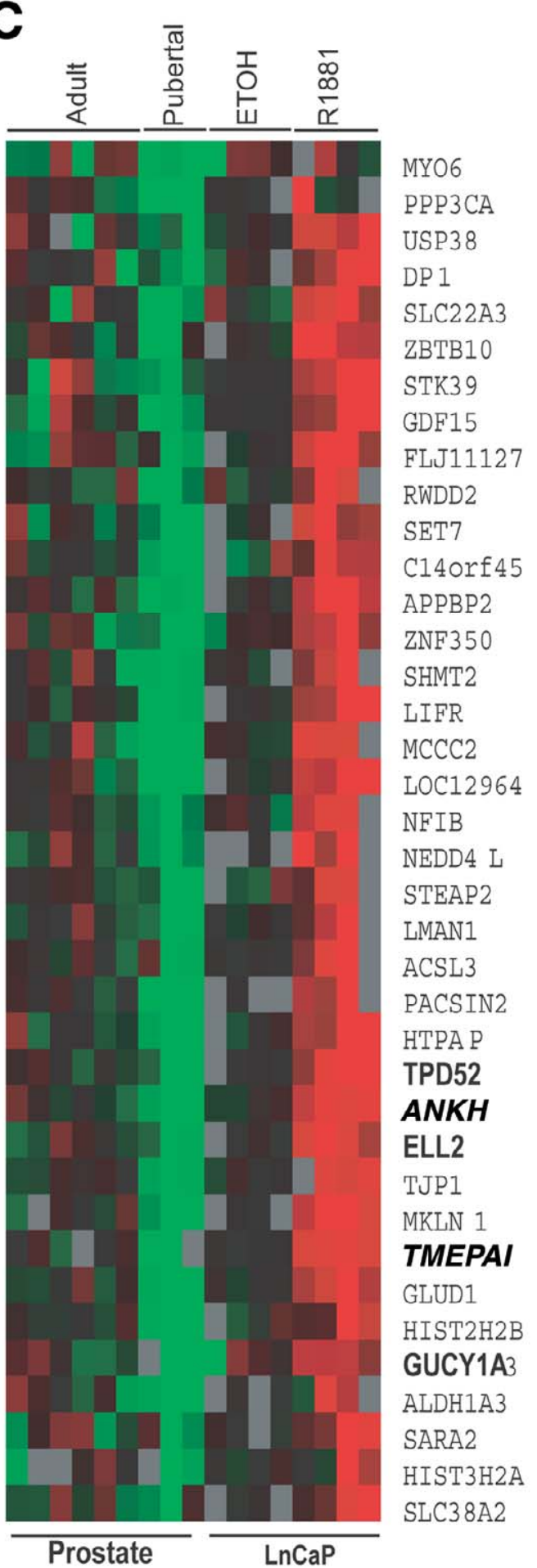

Figure 4. Gene expression meta-analysis to identify putative androgen target genes. $A$ ) A high correspondence in shared genes upregulated in $\mathrm{LNCaP}$ dataset and down-regulated in pubertal prostate. Black line indicates observed overlap and gray line shows expected overlap by probability between the datasets. $\boldsymbol{B}$ ) Statistical significance of the shared gene overlap. Arrow indicates nominal significance level at $P<0.05$. $C$ ) Expression pattern of 40 genes shared between top 300 pubertal genes (underexpressed in pubertal vs. adult prostate with mean-centered average less than -0.45) and the top 300 LNCaP androgen genes (overexpressed in R1881-treated cells with mean-centered average $>2$ ). Genes highlighted in bold appear in Table 2, and those italicized appear in Fig. 5. 
Fig. 5
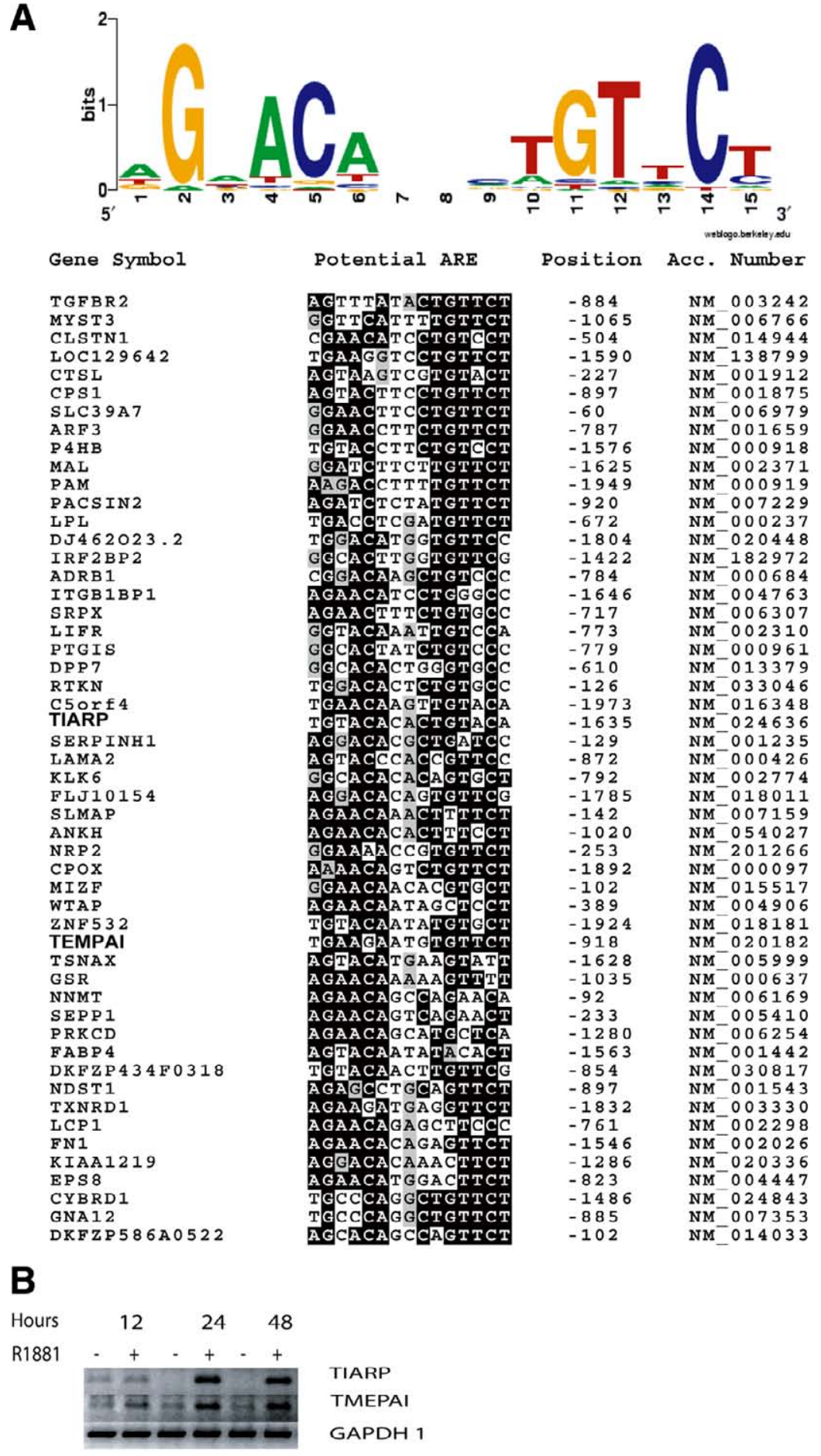

Figure 5. Identification of ARE motifs and androgen induction of TIARP. A) Putative human AREs identified by searching the $5^{\prime}$ regulatory regions of genes that are differentially regulated in pubertal prostate for a motif corresponding to the TRANSFAC ARE consensus sequence. A CLUSTALW alignment identifies highly conserved residues shaded in black. A consensus sequence generated by WEBLOGO displays frequency of each base in the consensus proportional to character height, with height of entire stack adjusted to signify information content of sequences at that position. B) RT-PCR analysis for TIARP and TMEAPI genes on RNA isolated from LNCaP cells treated with synthetic androgen R1881 across time points. - : Mock-treated cells; while +: androgen-treated cells. GAPDH amplification was used as an internal control. 
Fig. 6
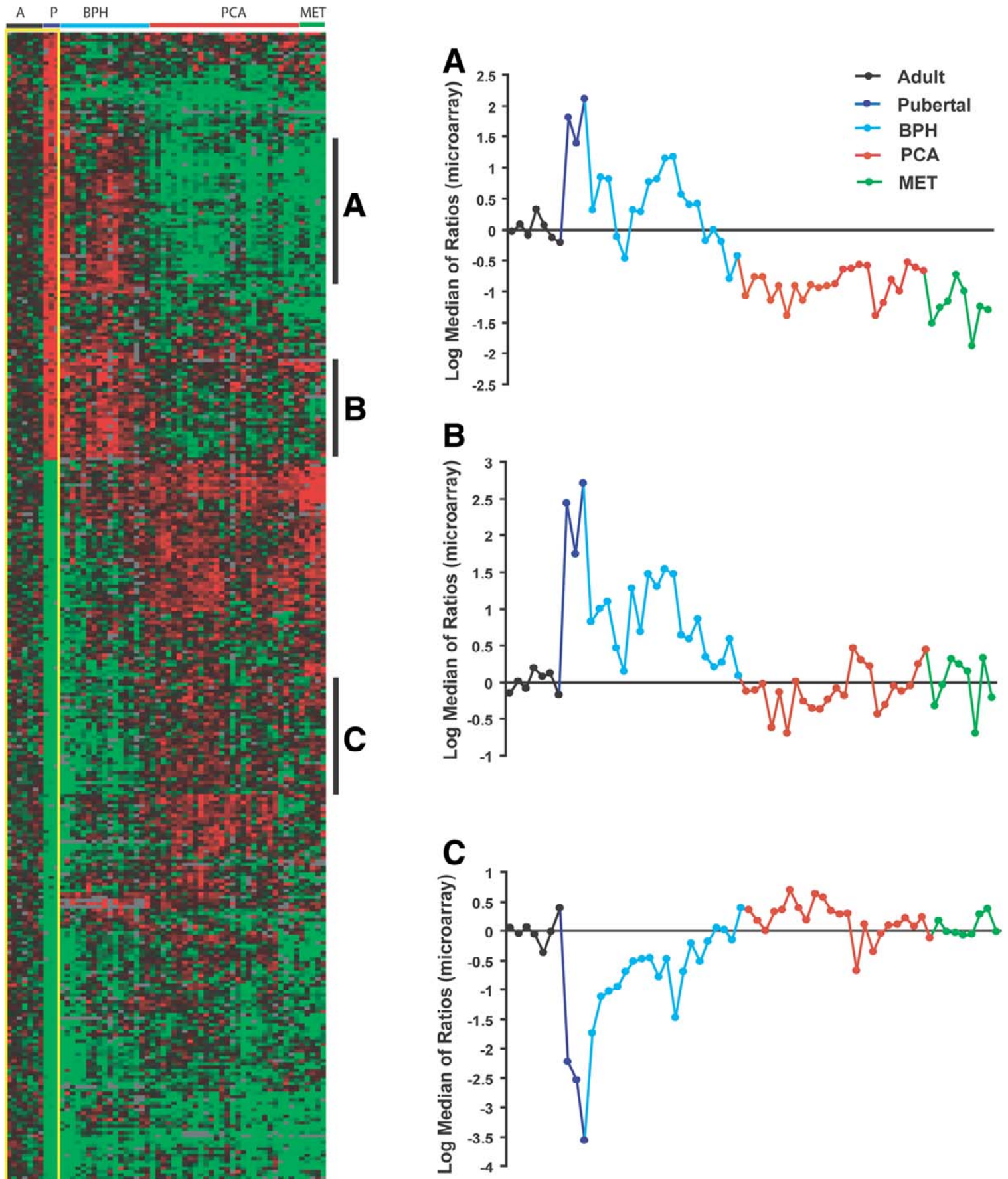

Figure 6. Comparison of pubertal prostate signature genes with other prostatic conditions. Gene expression signature of pubertal prostate compared with profiles obtained from various other disease states of the prostate namely benign prostatic hyperplasia (BPH), clinically localized prostate cancer (PCA), and hormone refractory-prostate cancer (MET). Regions of interest are highlighted including coordinately up-regulated $(\boldsymbol{A}, \boldsymbol{B})$ and down-regulated $(\boldsymbol{C})$ in both pubertal and BPH tissues. Graphs $\boldsymbol{A}, \boldsymbol{B}$, and $\boldsymbol{C}$ represent average normalized median of ratio values of genes that comprise the corresponding regions of interest $(\boldsymbol{A}, \boldsymbol{B}$, and $\boldsymbol{C})$ indicated in the treeview representation. Red indicates upregulated genes, green down-regulated genes, black no change, and gray data not available. 
Fig. 7

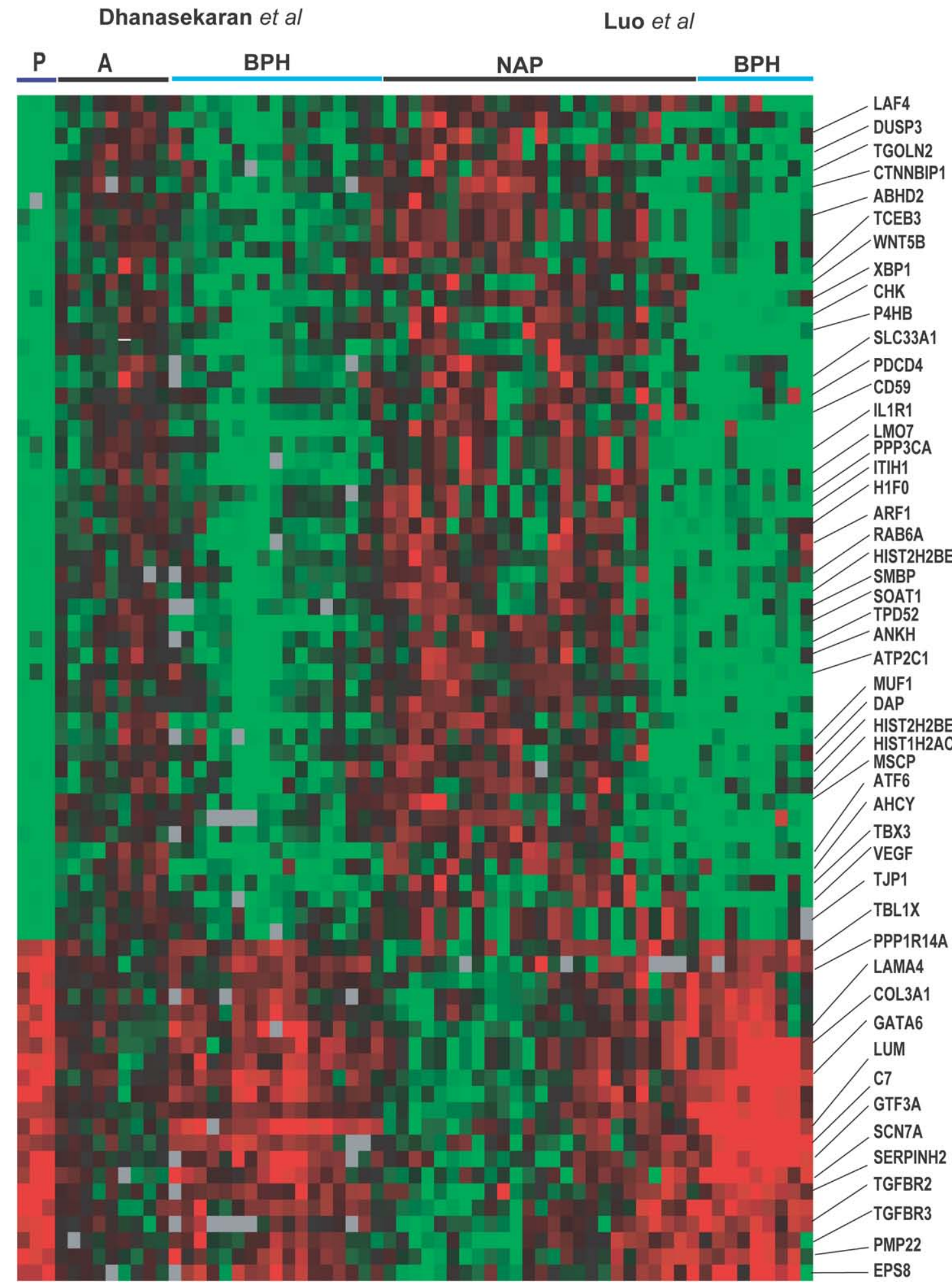

Figure 7. Gene expression pattern shared between pubertal prostate and BPH. Seventy three statistically significant genes that exhibited similar expression pattern in the pubertal prostate and 2 BPH datasets $(23,24)$. Red indicates upregulated genes, green down-regulated genes, black no change, and gray data not available. Gene symbols are provided (HUGO nomenclature). NAP: normal adjacent prostate; BPH: benign prostatic hyperplasia. 